\title{
The Categorical Organization of Semantic and Lexical Knowledge in the Brain
}

\author{
GUIDO GAINO'TTI
}

Institute of Neurology of the Catholic University of Rome, Policlinico Gemelli, Largo A. Gemelli, 8-00168 Roma, Italy

\begin{abstract}
In recent years several papers have shown that different verbal and non-verbal semantic categories can be selectively disrupted by brain damage and that consistent anatomical localizations correspond to each category-specific semantic disorder.

This paper aims to suggest that the brain regions typically damaged in a given type of category-specific semantic disorder might be critically involved in processing the kind of information which mainly contributes to organizing that semantic category and to distinguishing among its members.

This general hypothesis is discussed taking into account: (a) comprehension and production of object names (nouns) and of action names (verbs) in agrammatic and in anomic aphasic patients; (b) verbal and non-verbal identification of body parts; (c) verbal and non-verbal identification of living beings and of man made artefacts.
\end{abstract}

\section{Introduction}

The cerbral cortex is a very heterogeneous structure, characterized by an important division of labour among its various parts (see Hécaen and Lanteri-Laura, 1977 for survey).

Up until quite recently, however, it was generally held that this heterogeneity only concerned mechanisms subserving the different brain functions (i.e. the analysis of sensory information, the planning and execution of the motor response, the processing of various aspects of language and so on) but not different realms of knowledge acquired through these mechanisms. In the last ten years a selective impairment of different categories of knowledge, either in the semantic-lexical system or in modality-specific components of semantic memory has been demonstrated by the detailed neuropsychological study of single patients. Furthermore, an analysis of the parts of the cerebral cortex selectively damaged in these patients suggests that a certain relationship may exist between realms of semantic knowledge disrupted by the lesion and localization of brain mechanisms which could have critically contributed to their acquisition and storage.

In this review neuropsychological investigations which have demonstrated the selective impairment of a few well defined semantic categories, will be described and the following selective semantic disorder specifically

0953-4180/90/020109+07 $\$ 3.50 / 0 \quad$ (C) 1990 CNS (Clinical Neuroscience) Publishers 
discussed: (a) comprehension and production of object names (nouns) and of action names (verbs) in agrammatic and in anomic aphasic patients; (b) verbal and non-verbal identification of body parts; (c) verbal and nonverbal identification of living and non-living beings.

\section{Disorders in Production and Comprehension of Object Names and Action Names in Aphasia}

Since the late nineteen-sixties, clinical data have suggested that a different impairment of nouns and verbs occurs in anterior (non-fluent) and posterior (fluent) clinical forms of aphasia. Goodglass et al. (1966) noticed, for example, that fluent aphasics are more impaired in naming objects (producing nouns) than in naming actions (producing verbs), whereas non-fluent aphasics show the opposite pattern of impairment. This double dissociation was then further investigated focusing attention on agrammatic patients (within non-fluent aphasics) and on anomic patients (within fluent aphasics). Thus, Meyerson and Goodglass (1972), Marin et al. (1976) and Miceli et al. (1983) noticed that agrammatic patients tend to omit main (root) verbs, whereas Benson (1979) stressed the selective difficulty met by anomic patients in the selection and appropriate use of nouns. In group studies Miceli and coworkers have shown: (a) that agrammatic patients are more impaired in naming actions than objects, whereas anomic patients show the reverse pattern of impairment (Miceli et al., 1984); and that the same kind of dissociation can be observed in comprehension of nouns and verbs (Miceli $e t$ al., 1988).

In single case studies. McCarthy and Warrington (1985) have observed a selective impairment in comprehending and naming verbs in an agrammatic patient and have attributed this defect to a degradation of the semantic representation of the category "action names", whereas Zingeser and Berndt (1988) have described an anomic patient who presented a selective difficulty in producing nouns both in the oral and in the written modality.

The existence of a double dissociation between disruption of action names in anterior (agrammatic) patients and degradation of object names in posterior (anomic) patients is therefore well established. Tentative interpretations can be advanced to explain this double dissociation. The hypothesis that we suggest consists in searching in the anterior and in the posterior parts of the brain for some general mechanism which could play a critical role in the acquisition of the semantic representations of action names and, respectively of object names.

The well known dichotomy between the posterior parts of the brain which are substantially involved in operations of sensory analysis and the anterior parts which are mainly involved in operations of motor planning and of motor execution may be of relevance. This suggested to Geschwind a "neurological model" of naming, which seems much more appropriate for naming objects than for naming actions. According to Geschwind (1967) aphasic anomia results from damage to the angular gyrus (roughly placed at the core of the posterior part of the left hemisphere) because a large variety 
of sensory information converges in this region. This sensory convergence could supply the subject with the "attributes" necessary to arouse the verbal label corresponding to the semantic representation of the perceived object.

The Geschwind's model, originally proposed to explain every aspect of aphasic anomia, stressing the role of sensory attributes in establishing the semantic representation of objects, could, indeed, explain why anomic patients are particularly impaired in producing the comprehending object names. A similar model, stressing the importance of motor schemata in constructing the semantic representation of actions could explain why anterior (agrammatic) aphasic patients are more impaired in producing and comprehending action names than in naming objects and comprehending object names.

If this interpretation is correct we should, therefore, admit that the semantic representation of objects should chiefly rely upon the synthesis of their sensory attributes (processed by the posterior parts of the brain), whereas the semantic representation of actions would mainly result from the acquisition of motor schemata (elaborated by the anterior parts of the brain).

\section{Identification of Body Parts in Brain-Damaged Patients}

The hypothesis that body parts may have a special status within the various domains of semantic knowledge and that their identification may be selectively affected by brain injury, has been repeatedly raised during the history of neuropsychology.

The kind of impairment more frequently observed in a pure form in brain-damaged patients seems to concern the capacity to point propositionally to single parts of the body, both on verbal request and on imitation. This kind of neuropsychological impairment (which is usually labeled "autotopoagnosia") was first described by Pick (1908, 1922) and contributed to the development of popular (although fuzzy) concepts, such as those of "body schema" (Head, 1920) and of "body image" (Schilder, 1935). In more recent years, De Renzi and Faglioni (1963), De Renzi and Scotti (1970), Poncet et al. (1971) and Ogden (1985) have given detailed descriptions of patients presenting the following general characteristics (1) very mild language disorders, without important defects in naming or pointing to isolated parts of the body; (2) severe difficulties in finding single parts of their own body, of the body of the examiner or of a schematic model, both on verbal request and on imitation; (3) presence of an important lesion of the left parietal lobe. Some of these patients also presented similar difficulties in pointing to parts of complex objects, such as a bicycle or a car, but in at least one patient (Ogden, 1985) these disorders were quite specific for the human body, since the capacity to identify parts of complex objects or parts of animals' bodies was completely intact. Furthermore, a similar dissociation between a greater impairment in pointing to body parts and a better performance in pointing to parts of animals' bodies or to parts of instruments had already been observed in two 
patients by Assal and Buttet (1973). Taken together, all these data confirm the existence of a semantic representation of the human body, subserved by specific brain mechanisms and selectively disorganized by injuries encroaching upon the left parietal lobe. This impairment would selectively affect tasks based upon knowledge of the spatial relationships existing among the various parts of the human body. As for the basic mechanisms localized in the parietal lobe and involved in the construction of this semantic representation of the human body, they could be easily identified in somato-sensory integration mechanisms, housed in the anterior parts of the parietal lobe and always considered as the source of the raw data upon which the schematic representation of the human body could be constructed.

\section{Disorders in Identification of Living and Non-Living Beings in Brain-Damaged Patients}

The last group of category-specific semantic disturbances that we intend to discuss in this paper can be considered as the most surprising and the most recently discovered. It refers to the double dissociation between inability to recognize animals, flowers, fruits (and food), described by several authors in patients with herpes simplex encephalitis (Radcliff and Newcombe, 1982; Warrington and Shallice, 1984; Sartori and Job, 1988; Silveri and Gainotti, 1988) and a prevalent impairment in the identification of non-living things (vehicles, clothing, furniture and other kinds of manufactured articles described by Warrington and McCarthy $(1983,1987)$ ) in patients with extensive lesions of the left fronto-parietal cortex. The first of these category-specific semantic disorders is the most striking for at least three reasons: (1) the discrepancy between identification of living and non living beings is often very clear; (2) this category specific semantic impairment is often observed in patients showing no defect of language functions or of visual-perceptual analysis; (3) the same defect can be observed with similar characteristics in different modalities, using both verbal and non-verbal tasks.

Thus, if a patient is unable to name the picture of a banana, he is usually also unable to give a verbal description of the word "banana", to draw by memory a banana or to color in yellow the black and white picture of a banana. The only information that these patients are usually able to give concerns the superordinate category to which the living item belongs ("banana is a fruit"), but they generally fail to retrieve the attributes which typically define this item. This pattern of impaired and spared information has been attributed to a disruption of the structural description, i.e. to a loss of information concerning the figural characteristics of category members (Humphreys et al., 1988; Sartori and Job, 1988).

According to this interpretation, identification of living beings could be particularly difficult in these patients since identification of members belonging to different categories of living beings (such as animals, fruits, flowers and so on) may critically depend upon this structural description, i.e. subtle perceptual (and mainly visual) features. For example, the distinction 
between a strawberry, a blackberry and a raspberry crucially depends upon subtle differences in color, shape and taste of these different kinds of berries. A very different set of features are usually used to distinguish items belonging to various categories of manufactured articles (non-living items) since in this case functional information is much more relevant than perceptual attributes.

These arguments concerning the critical role played by perceptual (and mainly visual) attributes in the identification of living beings and, respectively, by functional features in the identification of inanimate objects (and above all of man made articles) could, perhaps, allow us to establish a link between the semantic categories selectively disrupted by the lesion, the localization of the underlying brain injury and the brain mechanisms which could have critically contributed to the acquisition of the disrupted semantic category. The anatomical localization of lesions provoking a. categoryspecific semantic disturbance for living beings seems rather typical, since almost all instances of this disorder reported in the neuropsychological literature had made a partial recovery from herpes simplex encephalitis. Now, since this disease typically impairs the temporo-limbic structures of both hemispheres (Esiri, 1982; Damasio and Van Hoesen, 1985; Bakchine et al., 1986) these structures should house a mechanism critically involved in the acquisition of high level visual information necessary to correctly identify different categories of living beings. This hypothesis is supported by the fact that bilateral removal of the temporal lobes provokes both in animals (Klüver and Bucy, 1939) and in man (Lilly et al., 1983; Bakchine et al., 1986) a disorder of visual recognition, which has been exhaustively investigated by Mishkin and coworkers (Mishkin, 1982; Mishkin and Appenzeller, 1987). According to these authors, this form of "visual agnosia" is due to disruption of functions of identification, learning and assignment of biological significance to complex visual stimuli, mainly subserved by amygdala and hippocampus.

If we pass, now to the localization of brain injuries which preferentially impair identification of non-living things (i.e. of man made artefacts) we see that these lesions severely damage the fronto-parietal regions of the left hemisphere (Warrington and McCarthy, 1983, 1987). A possible link between the anatomical locus of lesions producing this prevalent impairment of non-living things and the neurophysiological mechanisms involved in the acquisition of these categories of knowledge could consist in the fact that in the fronto-parietal areas of the brain are mainly represented somatosensory and motor functions.

It is, therefore, possible to assume that the categories of man made articles could be chiefly subserved by the fronto-parietal regions of the brain because knowledge of these categories is at least in part based upon handling, manual use, or, in any case, physical contact and concrete utilization of these objects.

This interpretation is also supported by the observation of Warrington and McCarthy (1987) that knowledge of small manipulable objects was more impaired in their patient than identification of large "out-door" 
objects. It must be acknowledged, on the other hand, that the dissociation between identification of living and non-living beings is much less impressive in these patients than in those presenting a category-specific semantic impairment for living beings. Furthermore, since both patients reported by Warrington and McCarthy $(1983,1987)$ presented a global aphasia, it is perhaps more correct to say that identification of living beings was in them relatively spared, rather than saying that identification of man made artefacts was selectively impaired.

The views expressed in this paper have the status of a preliminary very tentative hypothesis and must be considered with caution, to avoid the risk of premature, naive generalizations. However, these viewpoints are well in line with some basic assumptions of contemporary connectionistic models (Ballard, 1986; Churchland and Sejnowski, 1988). According to these models, information processing and storage are not separated, but closely intertwined in a network, since information is stored as a pattern of activity in the connections between the units of the network processing the same information. They hypothesis that the representations of different semantic categories might be closely intertwined with the neurophysiological mechanisms which have critically contributed to their acquisition seems, therefore, well in line with these basic assumptions and needs to be tested with further, more rigorous, prospective investigations.

\section{References}

Assal, G. and Buttier, J. (1973). Troubles du schéma corporel lors des atteintes hemisphèriques gauches. Praxis, Bern, 62, 172-179.

Bakchine, S., Chain, F. and Lhermitte, F. (1986). Syndrome de Klüver-Bucy humain complet après une encéphalite a herpès simplex type 2. Revue Neurologique (Paris), 142, 126-132.

Ballard, D. H. (1986). Cortical connections and parallel processing: structure and function. Behavioral and Brain Sciences, 9, 67-120.

Benson, F. (1979). Neurologic correlates of anomia. In "Studies in Neurolinguistics", H. Whitaker and H. A. Whitaker (Eds.) Vol. 4, Academic Press, New York.

Churchland P. S. and Sejnowski, T. J. (1988). Neural representation and neural computation. In "Biological Computation" (Ed. L. Nadel) MIT Press, Cambridge.

Damasio, A. R. and Van Hoesen (1985). The limbic system and the localisation of herpes simplex encephalitis. Journal of Neurology, Neurosurgery and Psychiatry, 48, 297-301.

De Renzi, E. and Faglioni, P. (1963). L'autotopoagnosia. Archivio di Psicologia, Neurologia e Psichiatria, 24, 288-322.

De Renzi, E. and Scotti, G. (1970). Autopoagnosia: Fiction or reality. Archives of Neurology, 23, 221-227.

Esirir, M. M. (1982). Herpes simplex encephalitis: An immunohistological study of the distribution of viral antigen within the brain. Journal of Neurological Science, 54, 209-26.

Geschwind, N. (1967). The varieties of naming errors. Cortex, 3, 97-112.

Goodglass, H., Klein, B., Carey, P. and Jones, K. (1966). Specific semantic word categories in aphasia. Cortex, 2, 74-89.

Head, H. (1920). "Studies in Neurology". Oxford University Press, London.

Hécaen, H. and Lanteri-Laura, G. (1977). "Evolution des Connaissances et des Doctrines sur les Localisations cérébrales." Desclée de Brouwer, Paris.

Humphreys, G. W., Riddoch, M. J. and Quinlan, P. T. (1988). Cascade processes in picture identification. Cognitive Neuropsychology, 5, 67-103. 
Klüver, H. and Bucy, P. C. (1939). Preliminary analysis of functions of the temporal lobes in monkeys. Archives of Neurology and Psychiatry, 42, 979-1000.

Lilly, R., Cummings, J. L., Benson, D. F. and Frankel, M. (1983). The human Klüver-Bucy syndrome. Neurology (Cleveland), 33, 1141-1145.

Marin, O. S. M., Saffran, E. M. and Schwartz, M. F. (1976). Dissociations of language in aphasia: Implications for normal functions. Annals of New York Academy of Sciences, 280, 868-884.

McCarthy, R. A. and Warrington, E. K. (1985). Category specificity in an agrammatic patient: the relative impairment of verb retrieval and comprehension. Neuropsychologia, 23, 709-727.

Miceli, G., Mazzucchi, A., Menn, L. and Goodglass, H. (1983). Contrasting cases of Italian agrammatic aphasia without comprehension disorder. Brain and Language, 19, 65-97.

Miceli, G., Silveri, M. C., Nocentini, U. and Caramazza, A. (1988). Patterns of dissociation in comprehension and production of nouns and verbs. Aphasiology, 2, 351-358.

Miceli, G., Silveri, M. C., Villa, G. and Caramazza, A. (1984). On the basis for the agrammatic's difficulty in producing main verbs. Cortex, 20, 207-220.

Mishkin, M. (1982). A memory system in the monkey. Philosophical Transaction of the Royal Society, London, 298, 85-89.

Mishkin, M. and Appenzeller, T. (1987). The anatomy of memory. Scientific American, 256, 80-89.

Myerson, R. and Goodglass, H. (1972). Transformational grammars of three agrammatic patients. Language and Speech, 15, 40-50.

Ogden, J. A. (1985). Autotopagnosia: Occurrence in a patient without nominal aphasia and with an intact ability to point to parts of animals and objects. Brain, 108, 1009-1022.

Pick, A. (1908). "Uber Storungen der Orientierung am eigenen Korper Arbeiten aus der deutschen psychiatrischen Universitats Klinik in Prag". Karger, Berlin. 1-19.

Pick, A. (1922). Storung der Orienterung am eigenen Korper, Beitrag zur Lehre vom Bewusstsein des eigenen Korpers. Psychologische Forschung, 1, 303-318.

Poncet, M., Pellissier, J. F., Sebahoun, M. and Nasser, C. J. (1971). A propos d'un cas d'autotopoagnosie secondaire à une lésion pariéto-occipitale de l'hémisphère majeur. Encéphale, 60, 110-123.

Ratcliff, G. and Newcombe, F. (1982). Object recognition: Some deductions from the clinical evidence. In "Normality and Pathology in Cognitive Functions" (Ed. A. W. Ellis). Academic Press, London.

Sartori, G. and Job, R. (1988). The oyster with four legs: a neuropsychological study of the interaction of visual and semantic information. Cognitive Neuropsychology, 5, 105-132.

Schilder, P. (1935). "The Image and Appearance of the Human Body". Kegan, London.

Silveri, M. C. and Gainotti, G. (1988). Interaction between vision and language in categoryspecific semantic impairment. Cognitive Neuropsychology, 5(6), 677-709.

Warrington, E. K. and McCarthy, R. (1983). Category specific access dysphasia. Brain, 106, 859-878.

Warrington, E. K. and McCarthy, R. A. (1987). Categories of knowledge: further fractionations and an attempted integration. Brain, 110, 1273-1296.

Warrington, E. K. and Shallice, T. (1984). Category specific and semantic impairments. Brain, 107, 829-853.

Zingeser, L. B. and Berndt, R. S. (1988). Grammatical class and context effects in a case of pure anomia: implications for models of language production. Cognitive Neuropsychology, 5(4), 473-516. 


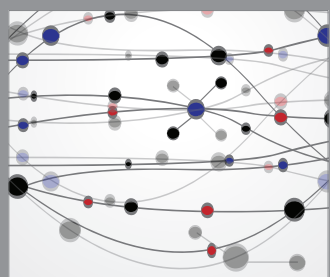

The Scientific World Journal
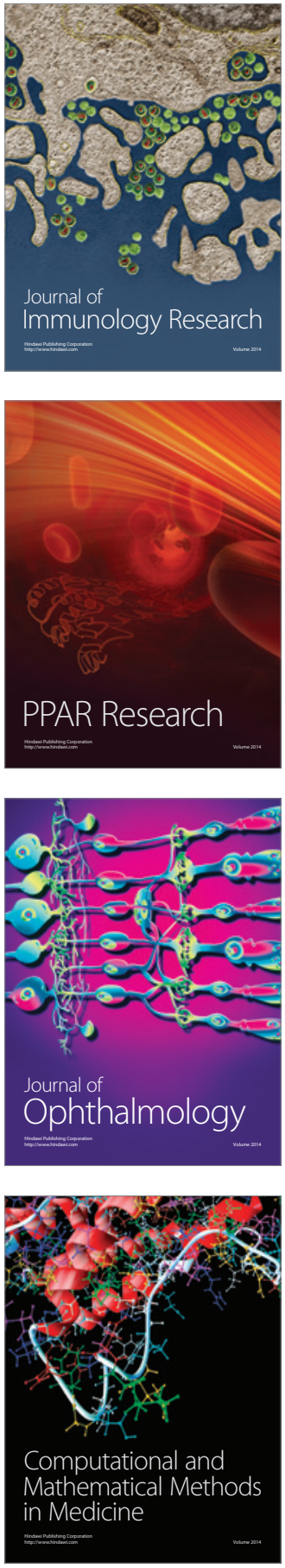

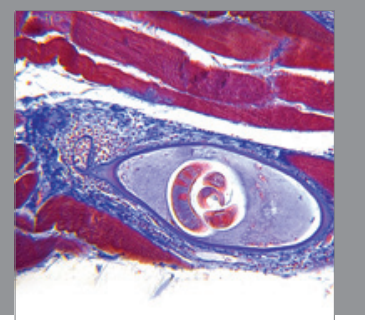

Gastroenterology

Research and Practice
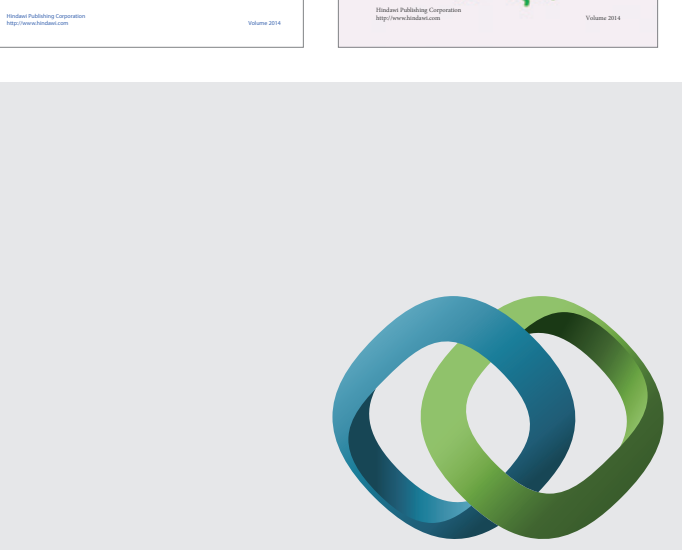

\section{Hindawi}

Submit your manuscripts at

http://www.hindawi.com
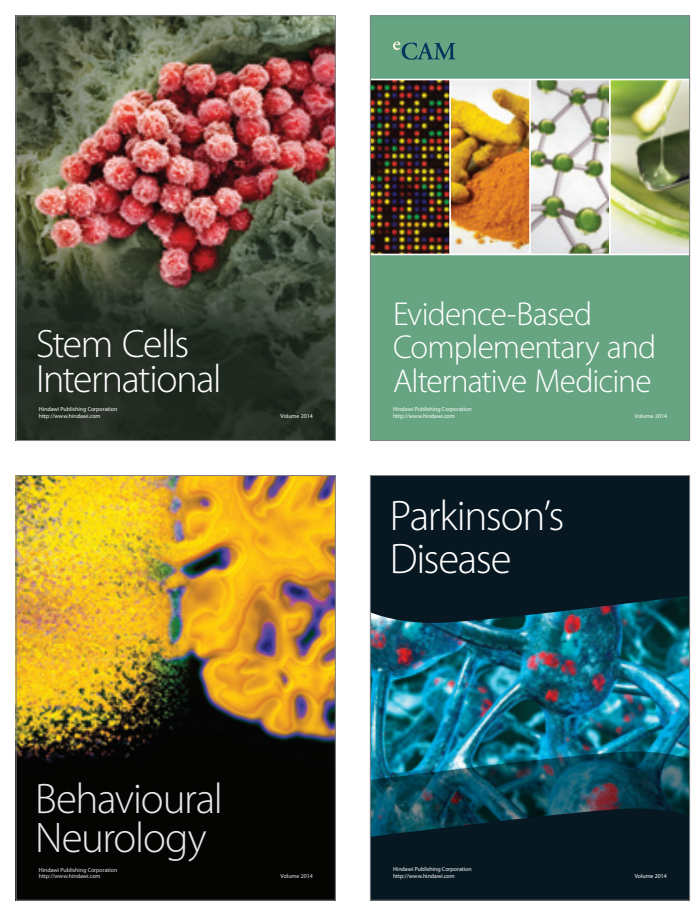

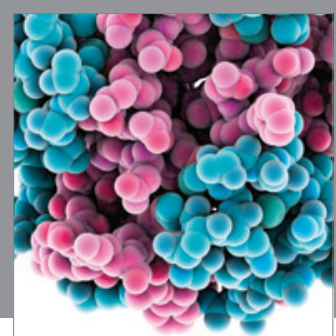

Journal of
Diabetes Research

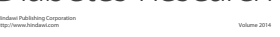

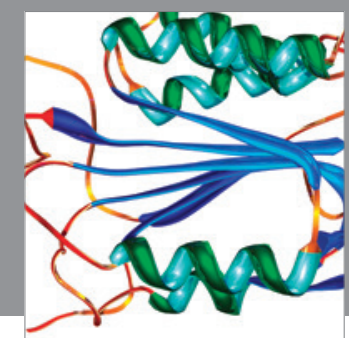

Disease Markers
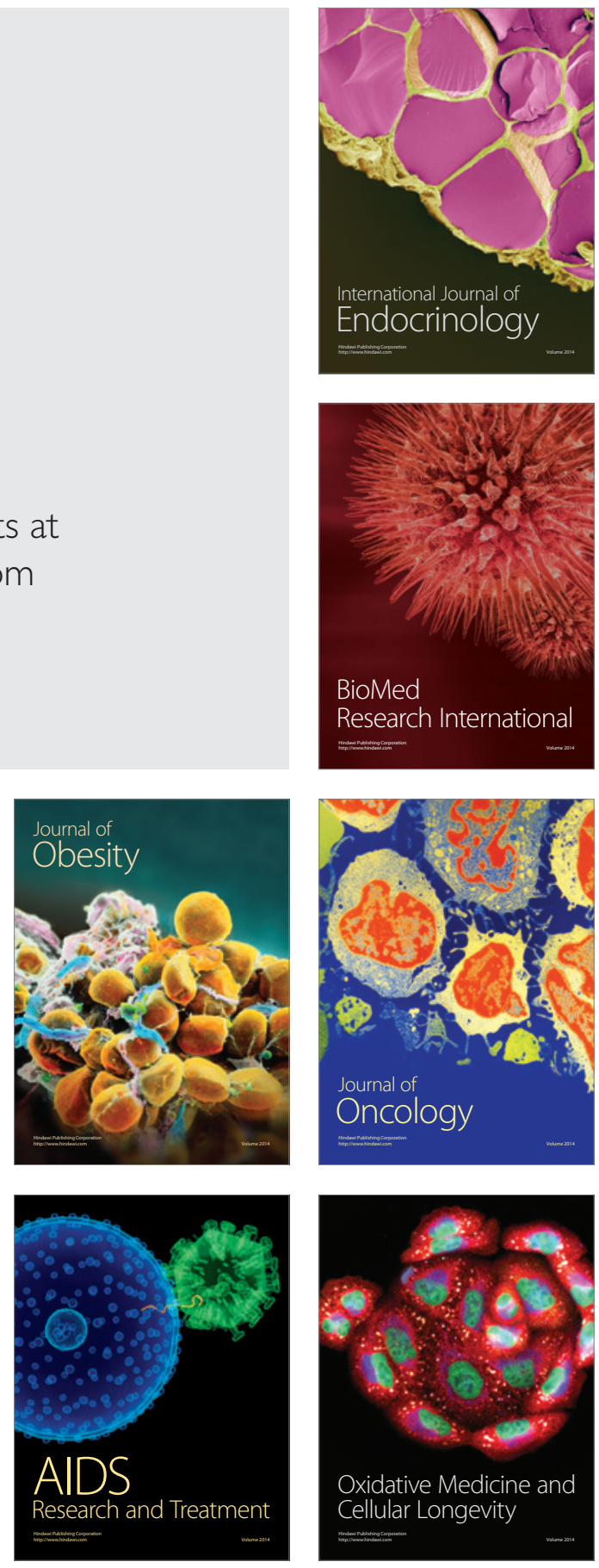